\title{
Primary Hyperoxaluria
}

National Cancer Institute

\section{Source}

National Cancer Institute. Primary Hyperoxaluria. NCI Thesaurus. Code C123158.

A hereditary disorder characterized by excessive oxalate production, leading to

hyperoxaluria. 